\title{
Short postoperative hospital stay after pancreaticoduodenectomy: what is real minimally invasive surgery?
}

\author{
Toshimi Kaido, Shuntaro Hirose, Yosuke Miyachi \\ Department of Gastroenterological and General Surgery, St. Luke's International Hospital, Tokyo, Japan \\ Correspondence to: Toshimi Kaido, MD, PhD. Department of Gastroenterological and General Surgery, St. Luke's International Hospital, 9-1 Akashi- \\ cho, Chuo-ku, Tokyo 104-8560, Japan. Email: kaido@luke.ac.jp.
}

Submitted Sep 30, 2021. Accepted for publication Oct 21, 2021.

doi: $10.21037 / \mathrm{hbsn}-21-410$

View this article at: https://dx.doi.org/10.21037/hbsn-21-410

Pancreaticoduodenectomy (PD) is performed for patients with tumors located in the region of the head of the pancreas, including cancer of the head of the pancreas, periampullary cancer, and distal bile duct cancer. PD is considered one of the most difficult and invasive surgeries in hepato-biliary-pancreatic surgeries because it requires meticulous techniques both in resection and reconstruction. Moreover, postoperative complications such as postoperative pancreatic fistula (POPF) and delayed gastric emptying (DGE) sometimes occur after PD, which leads to relatively higher mortality and longer postoperative hospital stay compared with other digestive surgeries. In $\mathrm{PD}$, laparoscopic and robotic surgeries, called minimally invasive surgery (MIS), have recently been introduced. However, the incidence of postoperative complications and the length of the postoperative hospital stay have not decreased significantly after the introduction of such MIS (1-3).

In East Asia, the average postoperative hospital stay after PD is around 3 weeks. Despite the recent introduction of the enhanced recovery after surgery (ERAS) program to hepato-biliary-pancreatic surgeries, the postoperative hospital stay after PD is still longer than after other hepatobiliary-pancreatic surgeries such as hemihepatectomy and distal pancreatectomy.

Peter Drucker [1909-2005], known as the father of management, emphasized the importance of marketing and innovation in the business world. This idea definitely seems to be applicable to the medical field. If we focus on the long postoperative hospital stay after $\mathrm{PD}$, we should consider the reasons why the postoperative hospital stay is long in PD. First, postoperative complications such as POPF and
DGE, which need drainage and sometimes fasting, prevent early discharge from hospital. Second, the application of the ERAS program to PD is still insufficient. Third, many patients for whom PD is planned are malnourished and have sarcopenia. We thought that if we could resolve these issues, the postoperative hospital stay would be naturally shortened. In this editorial, we would like to introduce our efforts to shorten the postoperative hospital stay after PD and our latest results. Moreover, we would like to discuss what real MIS is.

Our efforts to shorten postoperative hospital stay after PD can be divided into three parts: preoperative, intraoperative, and postoperative. We think that assessment and intervention are basic principles in every field, including perioperative management in PD. Preoperatively, we routinely assess patients' muscle strength and nutritional condition. We reported that preoperative sarcopenia was closely related to outcomes after hepato-biliary-pancreatic surgeries including hepatectomy and PD (4-9). Skeletal muscle mass (SMM) is usually evaluated by cross-sectional imaging such as computed tomography (CT) or magnetic resonance imaging (MRI) and a body composition analyzer, such as bioelectrical impedance analysis (BIA). Interestingly, both the European Working Group on Sarcopenia in Older People and the Asian Working Group for Sarcopenia most recently revised the sarcopenia diagnostic algorithm in 2019 and recommended to first measure muscle strength for early identification of people with, or at risk for, sarcopenia $(10,11)$. In clinical settings, measurement of hand grip strength (HGS) is simpler, as well as more cost-effective, than evaluation of SMM using CT, MRI, and BIA. We also reported that there was a significant strong positive 
correlation between SMM and HGS (12). Therefore, we consider that HGS can be a surrogate marker of SMM. Based on this idea, we started to assess HGS in patients planned for PD after April 2020. If HGS is less than $28.0 \mathrm{~kg}$ for men and less than $18.0 \mathrm{~kg}$ for women, patients undergo aggressive exercise intervention by rehabilitation staff in addition to a self-exercise training program at home. As for nutritional assessment, we focused on prealbumin (PA) and zinc among the various nutritional parameters. $\mathrm{PA}$, or transthyretin, is one of the rapid turnover proteins with a short (1.9 days) half-life. We started a nutritional intervention using a nutritional formula for patients with a serum PA level less than $15 \mathrm{mg} / \mathrm{dL}$. Zinc, an essential trace element, plays various pivotal roles in the human body and is needed for the activation of more than 300 enzymes. Serum zinc deficiency is known to induce impaired wound healing, appetite loss, and deterioration of immune function $(13,14)$, which would prevent postoperative early recovery. Therefore, we give oral zinc tablets to patients with a serum zinc level less than $80 \mu \mathrm{g} / \mathrm{dL}$.

Intraoperatively, we perform meticulous surgery to prevent postoperative complications with the idea that we can shorten postoperative hospital stay if major postoperative complications do not occur, especially in reconstruction. Our standard PD is open subtotal stomach-preserving PD. We prefer the modified Child reconstruction. For example, we clearly established our stent placement policy in pancreatico-jejunostomy (modified Blumgart's method): no stent for a hard pancreas with main pancreatic duct (MPD) diameter equal to or more than $7 \mathrm{~mm}$; placement of a 4-Fr tube into the MPD for external incomplete pancreatic juice drainage for a soft pancreas with an MPD diameter equal to or less than $3 \mathrm{~mm}$; and a lost stent for the other conditions. As for prevention of DGE, we perform gastro-jejunostomy using an automatic suture device to create an oblique anastomosis by elevating the afferent jejunal loop side. Moreover, we place the gastrojejunostomy to the left abdomen to enable straight flow from the stomach to the efferent jejunal loop.

Postoperatively, early mobilization, early oral intake, and early drain removal are essential. Patients start ambulation and drink clear water at 9 am on postoperative day (POD) 1 . After confirmation of the absence of DGE by upper gastrography on POD 3, patients take their first meal with nutritional guidance by dietitians. As for drainage, we usually place two drains into the foramen of Winslow and the dorsal side of the pancreatico-jejunostomy. We daily check the color, amount, and pancreatic amylase level of the drainage fluid from each drain. In principle, we remove each drain if the pancreatic amylase level of the drainage fluid of each one decreases to less than 1,000 U/L. We perform blood biochemical examinations on PODs 1,3 , and 5 and CT on POD 6. If there are no significant abnormal findings and patients can eat a moderate meal, the patients can be discharged from the hospital.

We have implemented the above policy since April 2020 and retrospectively examined its validity. The study protocol was approved by the Ethics Committee of St Luke's International Hospital (approval number 20-R104) and was conducted in accordance with the Declaration of Helsinki (as revised in 2013). Twenty-one consecutive patients who underwent open PD with curative intent by July 2021 were enrolled. The patients' characteristics and intraoperative findings are shown in Table 1. Five of 21 patients (24\%) were over 80 years old. The preoperative diagnosis was cancer of the head of the pancreas in 9, distal bile duct cancer in 6 , periampullary cancer in 2 , and so on. The median operative time was $496 \mathrm{~min}$, and median operative blood loss was $380 \mathrm{~mL}$.

Postoperative outcomes are shown in Table 2. Grade B POPF was found in 2 patients (10\%). No DGE was found. The median day of drain removal was POD 3. The median postoperative hospital stay was 8 days, ranging from 6 to 26 days. All patients returned to their own homes. Only 1 patient was readmitted to our hospital within 2 weeks after surgery due to appetite loss. These findings show that our new protocol, which consists of appropriate preoperative intervention (exercise and nutritional intervention based on accurate assessment), perioperative management considering ERAS, and performance of meticulous surgery with less complications could definitely achieve early discharge from hospital after PD.

Today, laparoscopic and robotic surgeries are called MIS worldwide, because these surgeries are considered to be less invasive in terms of their short-wound length. Recently, such MIS is being enthusiastically performed in various kinds of surgeries including $\mathrm{PD}$. We have also introduced these surgeries in our department. However, we should bear in mind that performance of MIS should not be the objective, but one of the modalities. If not safely performed, such MIS can cause severe postoperative complications leading to a long postoperative hospital stay or, if uncontrolled, death. As for PD, we have not yet introduced such "MIS". If the postoperative hospital stay is 3 weeks or longer after laparoscopic or robotic PD with or without major postoperative complications, should it really 
Table 1 Characteristics of patients

\begin{tabular}{|c|c|}
\hline Characteristic & Data \\
\hline Age (y) & 68 [46-84] \\
\hline \multicolumn{2}{|l|}{ Sex } \\
\hline Male/female & $14 / 7$ \\
\hline \multicolumn{2}{|l|}{ Original disease } \\
\hline Pancreas head cancer & $9(43.0)$ \\
\hline Distal bile duct cancer & $6(29.0)$ \\
\hline Ampullary cancer & $2(10.0)$ \\
\hline Pancreas body cancer & $1(5.0)$ \\
\hline IPMN & $1(5.0)$ \\
\hline Gastrinoma & $1(5.0)$ \\
\hline Solitary fibrous tumor & $1(5.0)$ \\
\hline \multicolumn{2}{|l|}{ Operative procedure } \\
\hline SSPPD/PD & $19 / 2$ \\
\hline Operative time (min) & 496 [429-719] \\
\hline Blood loss (mL) & $380[70-1,155]$ \\
\hline \multicolumn{2}{|l|}{ Pancreatic texture } \\
\hline Soft/hard & $10 / 11$ \\
\hline \multicolumn{2}{|l|}{ Stent tube into MPD } \\
\hline No/lost/incomplete & $5 / 11 / 5$ \\
\hline
\end{tabular}

Data are presented as $\mathrm{n}(\%)$ or median [range]. IPMN, intraductal papillary mucinous neoplasm; SSPPD, substomach preserving pancreaticoduodenectomy; PD, pancreaticoduodenectomy; MPD, main pancreatic duct.

Table 2 Postoperative outcomes

\begin{tabular}{lc}
\hline Outcome & Data \\
\hline First ambulation (POD) & $1[1-2]$ \\
First meal (POD) & $4[3-4]$ \\
Drain removal (POD) & $3[2-25]$ \\
Postoperative complications & $2 / 0$ \\
POPF (grade B/C) & 0 \\
Delayed gastric emptying & $8[6-26]$ \\
Postoperative hospital stay (day) & 1
\end{tabular}

Data are presented as $\mathrm{n}$ or median [range]. POD, postoperative hospital stay; POPF, postoperative pancreatic fistula. be called MIS? Although all patients underwent PD by open surgery in this cohort, we can surely say that early discharge from hospital at around 8 days would be physically and economically less invasive for patients. We think that this is "real MIS". In other words, whether a procedure is real MIS should be judged by outcomes, not by the surgical modality.

Once again, we should bear in mind that performance of MIS should not be the objective, but one of the modalities. We should select the appropriate surgical modality for each patient considering the conditions of the patient and the tumor. In conclusion, "real MIS" is not surgery performed by laparoscopic or robotic surgery, but surgery that provides good outcomes with early recovery for patients, irrespective of the surgical modality. To achieve such "real MIS", adequate preoperative intervention, perioperative management, and meticulous surgery are mandatory.

\section{Acknowledgments}

Funding: None.

\section{Footnote}

Provenance and Peer Review: This article was commissioned by the editorial office, Hepatobiliary Surgery and Nutrition. The article did not undergo external peer review.

Conflicts of Interest: All authors have completed the ICMJE uniform disclosure form (available at https:// hbsn.amegroups.com/article/view/10.21037/hbsn-21$410 /$ coif). Dr. TK serves as an unpaid editorial board member of Hepatobiliary Surgery and Nutrition. The other authors have no conflicts of interest to declare.

Ethical Statement: The authors are accountable for all aspects of the work in ensuring that questions related to the accuracy or integrity of any part of the work are appropriately investigated and resolved.

Open Access Statement: This is an Open Access article distributed in accordance with the Creative Commons Attribution-NonCommercial-NoDerivs 4.0 International License (CC BY-NC-ND 4.0), which permits the non- 
commercial replication and distribution of the article with the strict proviso that no changes or edits are made and the original work is properly cited (including links to both the formal publication through the relevant DOI and the license). See: https://creativecommons.org/licenses/by-nc-nd/4.0/.

\section{References}

1. Nickel F, Haney CM, Kowalewski KF, et al. Laparoscopic Versus Open Pancreaticoduodenectomy: A Systematic Review and Meta-analysis of Randomized Controlled Trials. Ann Surg 2020;271:54-66.

2. Kamarajah SK, Bundred J, Marc OS, et al. Robotic versus conventional laparoscopic pancreaticoduodenectomy a systematic review and meta-analysis. Eur J Surg Oncol 2020;46:6-14.

3. Shin SH, Kim YJ, Song KB, et al. Totally laparoscopic or robot-assisted pancreaticoduodenectomy versus open surgery for periampullary neoplasms: separate systematic reviews and meta-analyses. Surg Endosc 2017;31:3459-74.

4. Kaido T, Ogawa K, Fujimoto Y, et al. Impact of sarcopenia on survival in patients undergoing living donor liver transplantation. Am J Transplant 2013;13:1549-56.

5. Hamaguchi Y, Kaido T, Okumura S, et al. Preoperative Visceral Adiposity and Muscularity Predict Poor Outcomes after Hepatectomy for Hepatocellular Carcinoma. Liver Cancer 2019;8:92-109.

6. Kobayashi A, Kaido T, Hamaguchi Y, et al. Impact of Sarcopenic Obesity on Outcomes in Patients Undergoing Hepatectomy for Hepatocellular Carcinoma. Ann Surg

Cite this article as: Kaido T, Hirose S, Miyachi Y. Short postoperative hospital stay after pancreaticoduodenectomy: what is real minimally invasive surgery? HepatoBiliary Surg Nutr 2021;10(6):853-856. doi: 10.21037/hbsn-21-410
2019;269:924-31.

7. Okumura S, Kaido T, Hamaguchi Y, et al. Impact of the preoperative quantity and quality of skeletal muscle on outcomes after resection of extrahepatic biliary malignancies. Surgery 2016;159:821-33.

8. Okumura S, Kaido T, Hamaguchi Y, et al. Impact of Skeletal Muscle Mass, Muscle Quality, and Visceral Adiposity on Outcomes Following Resection of Intrahepatic Cholangiocarcinoma. Ann Surg Oncol 2017;24:1037-45.

9. Okumura S, Kaido T, Hamaguchi Y, et al. Visceral Adiposity and Sarcopenic Visceral Obesity are Associated with Poor Prognosis After Resection of Pancreatic Cancer. Ann Surg Oncol 2017;24:3732-40.

10. Cruz-Jentoft AJ, Bahat G, Bauer J, et al. Sarcopenia: revised European consensus on definition and diagnosis. Age Ageing 2019;48:16-31.

11. Chen LK, Woo J, Assantachai P, et al. Asian Working Group for Sarcopenia: 2019 Consensus Update on Sarcopenia Diagnosis and Treatment. J Am Med Dir Assoc 2020;21:300-307.e2.

12. Kaido T, Tamai $Y$, Hamaguchi $Y$, et al. Effects of pretransplant sarcopenia and sequential changes in sarcopenic parameters after living donor liver transplantation. Nutrition 2017;33:195-8.

13. Kogan S, Sood A, Garnick MS. Zinc and Wound Healing: A Review of Zinc Physiology and Clinical Applications. Wounds 2017;29:102-6.

14. Gammoh NZ, Rink L. Zinc in Infection and Inflammation. Nutrients 2017;9:624. 